\title{
A NEW FRAGMENT OF THE ALTUN YARUK IN OLD UIGUR FROM TUYUK
}

\author{
ZHANG TIESHAN \\ Academy for Research on Chinese Ethnic Minority Languages of Minzu University of China \\ 27 Zhongguancun South Avenue Haidian District, Beijing,100081, P. R. China \\ e-mail: zhangtieshan@sina.com
}

The fragment we are publishing here is preserved in the Academia Turfanica. It belongs to the second chapter of the first volume of the Altun Yaruk Sudur in old Uigur. There are some words that are apparently different from the other version(s) of the text.

Key words: Old Uigur, Altun Yaruk Sudur, Tuyuq.

\section{Introduction}

The new fragment we are publishing here ${ }^{1}$ was found on the east side of the Tuyuq caves in 2011 and is now preserved in the Academia Turfanica (吐魯番研究院) of Xinjiang. Its shelf number is TLF 14894 . The size of the fragment is $18 \times 29 \mathrm{~cm}$. The lines containing the title "altun önglüg yrü̈ yaltriqli $\ddot{q}$ opda kötrülmiš nom il[ig]i

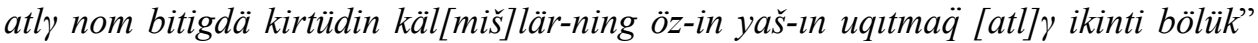
(recto 07 to 12) are written in red ink.

There are some parallels among the fragments belonging to the second chapter (first volume) of the old Uigur translation of the Suvarnaprabhāsasütra edited so far. ${ }^{2}$ The new leaf accomplishes the text in some parts.

\footnotetext{
${ }^{1}$ I would like to express my sincere thanks to Professor Peter Zieme who kindly checked my paper, but I am responsible for all mistakes.

${ }^{2}$ Cf. BT XVIII.
} 


\section{Transcription of the Old Uigur Text}

(recto)

01 [ym]ä ök äšidgäli bolur b[u] nom

02 [är]dinig

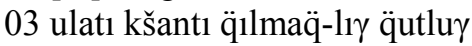

04 törüg ymä tip :

05 [t]ngri [tngr]isi burxan munčulayu

06 yrlı̈̆adı :

07 altun

önglüg

08 yruq̈ yaltrıq

-lı q̈opda

09 kötrülmiš nom il[ig]i

10 atl $\gamma$ nom bitigdä kirtüdin käl[miš]

11 lär-ning öz-in yaš-1n uqıtmaä

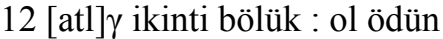

13 [

]d[a] bir somakitu atl $\gamma$

14 [bodistv

o]l bodistv ymä

15 [

ülgü]süz sansız

16[

17[

burx]an-lar

(verso)

00 baštınq̈1 üč [yigirmi p] tr

01 y[ir]tinčüdä ür kič uzun [

02 bkin mänggün turu yrlı̈̆amad[1]

03 inčip yänä nä ärsär kiši osu

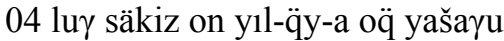

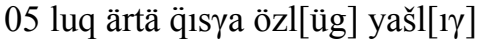

06 bolu yrliäa a luq : bu šu näng

07 šakimuni burxan-

08 nıng ädgü -singä

09 äd[räm]ingä o oršatı tip

10 [s] $]$ z sav ärmäz : nä üčün tip

11 tisär : q̈ltı tngri tngrisi burxan

12 oq̈ iki türlüg avant [tılta ]

13 üzä öz yaš ärti[ngü uzun bolur]

14 tip yrlı̈̈amıšı [

15 tip tisär ba[r

16 [ber]mäk [

17 [ 


\section{Translation of the Old Uigur Text}

(recto)

(01-04) And they will be able to listen to this dharma-sütra and also to the holy dharma of making confession.

(大正大藏經 T.XVII.665.404b26) 方得聞是經, 及以懺悔法。

(05-06) Thus the God of Gods Buddha was gracious.

This sentence is an additional phrase.

(Title of Chapter II)

(07-12) The second chapter called "Teaching the life (duration) of the Truly Come Ones" in the dharma scripture called "Most Magnificent Dharma King of Golden Light".

(大正大藏經 T.XVII.665.404b27) 金光明最勝王經如來壽量品第二

(12-17) At that time, [a Bodhisattva Mahāsattva] called Somaketu [lived in the city of Rājagrha]. This Bodhisattva [paid veneration to measure]less and numberless [Buddhas ....].

(大正大藏經 T.XVII.665.404b28) 爾時, 王舍大城有一菩薩摩訶薩，名曰 妙 (29) 幢, 已於過去無量俱胝那属多百千佛所, (404c01) 承事供養, 殖諸善 根。

(verso)

(00) (13th) leaf of the first volume

(The Bodhisattva Somaketu had this thought:) (01-06) [Why] did [the Buddha] not deign to stay a long (time) steady and forever in the world? Instead to deign to have only a very short life of just eighty years like any human being?

(07-16) This matter is not the right word equal to the virtues and manners of the Buddha Sākyamuni. Why? As said by the God of Gods Buddha, there are two kinds of reasons and [grounds] that life [will be] very long. [Which] are they? (...)

是時妙幢菩薩獨於 $(404 \mathrm{c} 02)$ 靜處, 作是思惟：「以何因緣, 釋迦牟尼如 來 $(404 \mathrm{c} 03)$ 壽命短促, 唯八十年?」復作是念：「如佛所說, $(404 \mathrm{c} 04)$ 有二 因緣, 得壽命長。云何為二?

\section{Notes on the Old Uigur Text}

(recto)

01-06 Parallel in BT XVIII, 0456-0457 [äši]dgäli bolur bo nom ärdini[g ulat1]

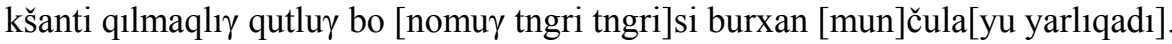


07-12 From the title only few letters likewise written in red ink are preserved in $\mathrm{U} 2567 .^{3}$

(verso)

There are parallel fragments for this part, too ${ }^{4}$, but they contain apparently a different version with some other words.

\section{Word Index}

$(\mathrm{r}=$ recto, $\mathrm{v}=$ verso $)$

\begin{tabular}{|c|c|c|c|}
\hline altun & r07 & käl-miš & $\mathrm{r} 10$ \\
\hline atl $\gamma$ & $\mathrm{r} 10, \mathrm{r} 12, \mathrm{r} 13$ & kirtü-din & r10 \\
\hline \multirow[t]{2}{*}{ avant } & v12 & kiši & v03 \\
\hline & & kötrül-miš & r09 \\
\hline ädgü-singä & v08 & kšant1 & r03 \\
\hline är-mäz & v10; är-sär v03 & & \\
\hline ärdäm-ingä & v09 & mänggün & v02 \\
\hline ärdini-g & r02 & munčulayu & $\mathrm{r} 05$ \\
\hline ärtä & $\mathrm{v} 05$ & & \\
\hline ärtingü & v13 & nä & v10 \\
\hline äšid-gäli & r01 & nom & $\mathrm{r} 01, \mathrm{r} 09, \mathrm{r} 10$ \\
\hline bar & v15 & oүšat1 & v09 \\
\hline baštın̈̈1 & $\mathrm{v} 00$ & ol & $\mathrm{r} 12, \mathrm{r} 14$ \\
\hline bkin & v02 & on & v04 \\
\hline ber-mäk & v16 & ö̈ & $\mathrm{v} 04, \mathrm{v} 12$ \\
\hline bir & r13 & osu $\gamma-1 u \gamma$ & $\mathrm{v} 03-04$ \\
\hline bitig-dä & r10 & & \\
\hline bodistv & r14 & ödün & $\mathrm{r} 12$ \\
\hline bol-ur & r01, v13; bol-u v06 & ök & $\mathrm{r} 01$ \\
\hline bölük & r12 & önglüg & r07 \\
\hline bu & r01, v06 & öz & v13; öz-in r11 \\
\hline \multirow[t]{2}{*}{ burxan } & $\begin{array}{l}\text { r05, v11; burxan-lar r16; } \\
\text { burxan-n1ng v07-08 }\end{array}$ & öz-lüg & v05 \\
\hline & & ptr & v00 \\
\hline ilig-i & r09 & & \\
\hline iki & v12 & q̈ltı & v11 \\
\hline ikinti & r12 & q̈1lmä̈-lı & $\mathrm{r} 03$ \\
\hline inčip & v03 & $\ddot{q}_{1 s \gamma a}$ & v05 \\
\hline
\end{tabular}




\begin{tabular}{|c|c|c|c|}
\hline q̈op-da & $\mathrm{r} 08$ & uq1tmä̈ & r11 \\
\hline q̈ut-luy & r03 & uzun & v01, v13 \\
\hline \multirow[t]{2}{*}{ q̈y-a } & v04 & & \\
\hline & & üč & v00 \\
\hline sans1z & $\mathrm{r} 15$ & üčün & v10 \\
\hline sav & v10 & ülgüsüz & r15 \\
\hline säkiz & v04 & ür kič & v01 \\
\hline somakitu & v13 & üzä & v13 \\
\hline \multirow[t]{2}{*}{ söz } & v10 & & \\
\hline & & yaltrıq-li $\gamma$ & r08 \\
\hline šakimuni & v07 & yrliqa-d1 & r06; yrlıqa-mad1 v02; \\
\hline$\check{s} \mathrm{u}$ & v06 & & $\begin{array}{l}\text { yrlıqamiš1 v14; yrlıqa- } \\
\text { yuluq v06 }\end{array}$ \\
\hline tngri & r05, v11; tngri-si r05, v11 & yruq̈ & r08 \\
\hline tilta & v12 & yaš & v13; yaš-1n r11 \\
\hline ti-p & $\begin{array}{l}\text { r04, v09, v10, v14, v15; ti- } \\
\text { sär v11, v15 }\end{array}$ & $\begin{array}{l}\text { yaša- } \gamma \text { uluq } \\
\text { yašlı } \gamma\end{array}$ & $\begin{array}{l}\mathrm{v} 05-06 \\
\mathrm{v} 05\end{array}$ \\
\hline törü-g & r04 & yänä & v03 \\
\hline tur-u & v02 & y1l & v04 \\
\hline türlüg & v12 & $\begin{array}{l}\text { ymä } \\
\text { yirtinčü }\end{array}$ & $\begin{array}{l}\mathrm{r} 01, \mathrm{r} 04, \mathrm{r} 14, \\
\mathrm{v} 01\end{array}$ \\
\hline ulat1 & $\mathrm{r} 03$ & {$[\mathrm{da}$} & r13 \\
\hline
\end{tabular}

\section{References}

BT XVIII = P. Zieme: Altun Yaruq Sudur. Vorworte und das Erste Buch. Edition und Übersetzung der alttürkischen Version des Goldglanzsūtra (Suvarnaprabhāsottama-sūtra). Turnhout, 1996 (Berliner Turfantexte XVIII).

Çagatay, S. (1945): Altun Yaruk'tan iki parça. Ankara.

Clauson, G. (1972): An Etymological Dictionary of Pre-Thirteenth-Century Turkish. Oxford.

Ehlers, G. (1990): Kurzfassungen buddhistischer Legenden im Alttürkischen. In: Laut. J. P. - Röhrborn, K. (eds): Buddhistische Erzählliteratur und Hagiographie in türkischer Überlieferung. Wiesbaden, pp. 1-14.

Ehlers, G. (1987): Alttürkische Handschriften. Teil 2: Das Goldglanzsūtra und der buddhistische Legendenzyklus Dásakarmapathāvadānamālā. Stuttgart (Verzeichnis der Orientalischen Handschriften in Deutschland XIII, 10).

Emmerick, R. E. (1990): The Sūtra of Golden Light. Being a Translation of the Suvarnabhāsottamasūtra. Oxford.

Kaya, C. (1994): Uygurca Altun Yaruk. Giriş, Metin ve Dizin. Ankara.

Radloff, W.-Malov, S. E. (1913-1917): Suvarṇaprabhāsa (sutra zolotogo bleska). Sanktpeterburg (Bibliotheca Buddhica 17).

Raschmann, S. (2007): Alttürkische Handschriften. Teil 13, Band XIII, 21. Stuttgart.

Sevortjan, E. V. (1978): Ėtimologičeskij slovar' tjurkskich jazykov. Moskva.

大正大藏經. Tokyo 1924-1935.

Zieme, P. (1977): Zu den Legenden im uigurischen Goldglanzsūtra. In: TUBA 1, pp. 149-156. 


\section{Photographs}

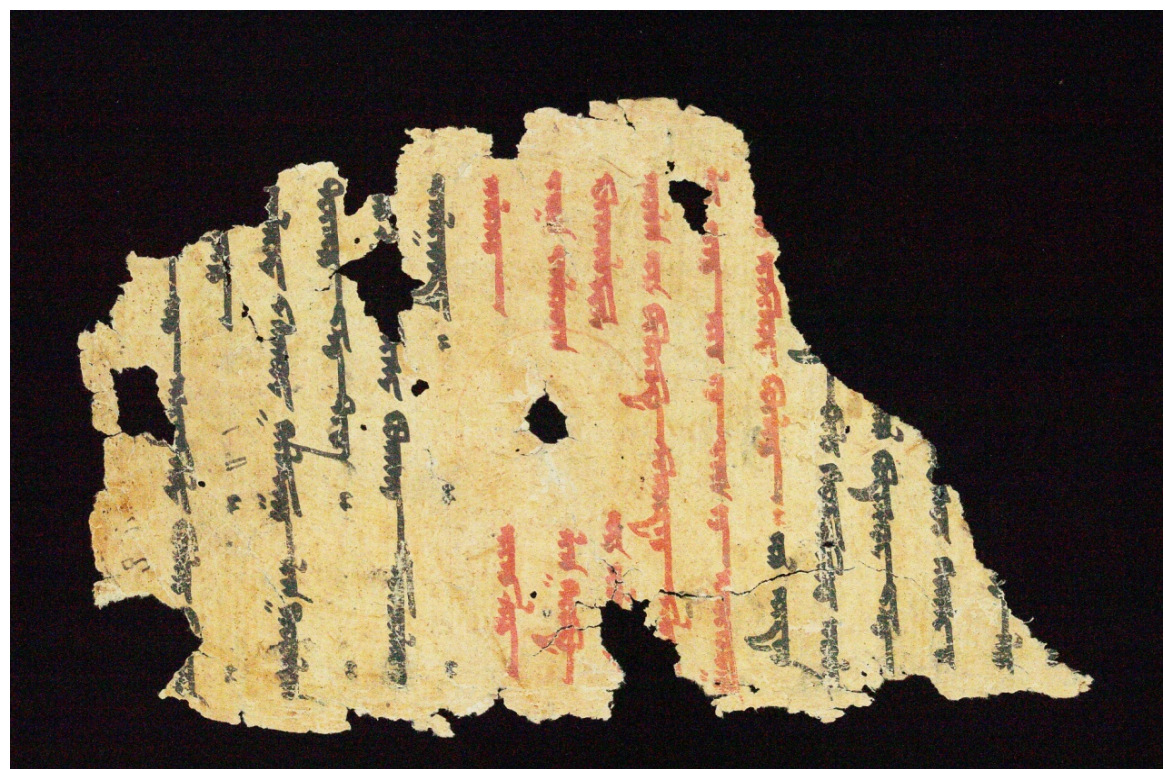

Figure 1. TLF 14894, recto

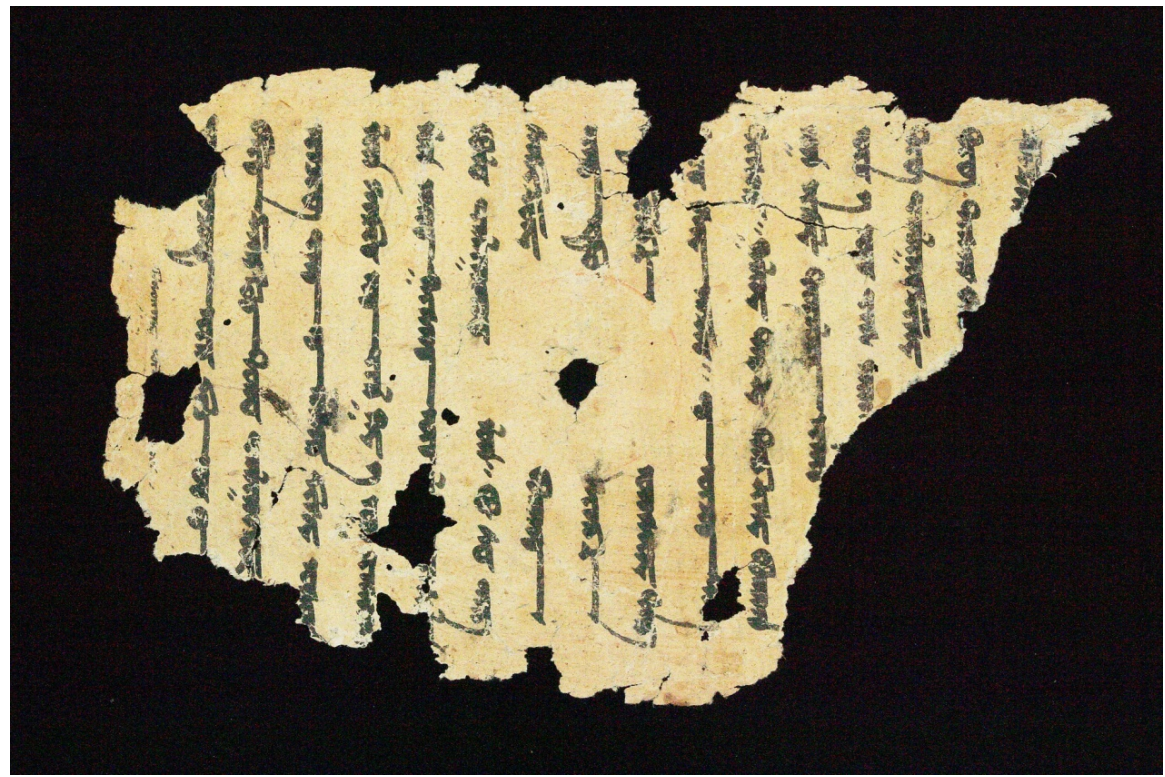

Figure 2. TLF 14894, verso 